\title{
A Case of Alveolar Rhabdomyosarcoma of the Ethmoid Sinus Invading the Orbit in an Adult
}

\author{
Hyun Seung Moon, MD, Sang Won Kwon, MD, Jong Hyeok Lee, MD \\ Department of Ophthalmology, Gachon Medical School, Gil Medical Center, Incheon, Korea
}

\begin{abstract}
Purpose: A case study and literature review of alveolar rhabdomyosarcoma (RMS) in an adult.
Methods: A 48-year-old male patient presented at our clinic complaining of proptosis that had persisted for 2 weeks in his left eye. A computed tomography (CT) scan revealed a destructive soft-tissue mass in the left ethmoid sinus with invasion of the left orbit and compression of the medial rectus muscle. Endoscopic intranasal biopsy revealed alveolar RMS. Conservative debulking and orbital wall decompression were performed.
\end{abstract}

Results: Immunohistochemical testing was positive for desmin, S-100, and smooth muscle actin (SMA), supporting the diagnosis of RMS. Since ipsilateral cervical and spinal metastasis was detected, systemic treatment was administered simultaneously.

Conclusions: Although rarely found in adults, RMS should be considered in the differential diagnosis of orbital tumors. Immunohistochemical analysis plays an important role in the definitive diagnosis of RMS.

Korean Journal of Ophthalmology 20(1):70-75, 2006

Key Words: Adult rhabdomyosarcoma, Ethmoid, Orbital tumor

Rhabdomyosarcoma (RMS) is the most common soft tissue sarcoma in the pediatric population, accounting for approximately $5 \%$ of all childhood cancers and about $20 \%$ of all malignant soft tissue tumors. ${ }^{1,2}$ The primary sites of RMS are: head and neck (40\%), genitourinary system $(20 \%)$, extremities (20\%), trunk (10\%), and other $(10 \%){ }^{3}$ Although RMS can occur at any age, it is primarily a disease of young children with a mean age at diagnosis of 8 years. Only a very small percentage of reported RMS cases have occurred in people over the age of 20 years. ${ }^{1,4}$ The present study describes a case of alveolar RMS of the ethmoid sinus invading the orbit in an adult and reviews related literature.

\section{Case Report}

A 48 year old male patient presented at our clinic complaining of proptosis and lid swelling in his left eye that had persisted for 2 weeks. The patient had no significant past

\footnotetext{
Received: September 15, 2005 Accepted: December 30, 2005

Reprint requests to Jong Hyeok Lee, MD. Department of Ophthalmology, Gachon Medical School, Gil Medical Center, \#1198, Guwol-dong, Namdong-gu, Incheon 405-760, Korea. Tel: 82-32-4603750, Fax: 82-32-460-3358, E-mail: ljhcyj@lycos.co.kr

* None of the authors has a financial or proprietary interest in any material or method mentioned

* This study was presented at the 93rd annual meeting of the Korean Ophthalmologic Society, April, 2005.
}

history or family history associated with ocular disease, trauma, or tumors. Although no palpable mass was noted, exophthalmometry measurements were $20 \mathrm{~mm}$ on the left, and $18 \mathrm{~mm}$ on the right with a base of $104 \mathrm{~mm}$. Motility was slightly limited in adduction. Results of ocular examination including visual acuity, color vision, and funduscopy were normal. The thyroid function test was also normal. A computed tomography (CT) scan revealed a destructive soft-tissue mass in the left ethmoid sinus with adjacent invasion of the left orbit and compression of the left medial rectus muscle (Fig. 1). Magnetic resonance imaging (MRI) showed that the signal intensity of the mass was low on a T1-weighted image with contrast enhancement and isointense to muscles on a $\mathrm{T} 2$-weighted image. The lesion extended into the left orbit with compression of the left medial rectus muscle, and there was also intracranial extension with compression of left side frontal lobe (Fig. 2).

A fiberoptic endoscopic biopsy of the nasal cavity revealed alveolar RMS. Metastatic work-up revealed cervical lymph node involvement, indicating a stage IV RMS based on the Intergroup Rhabdomyosarcoma Study (IRS) TNM Pretreatment Staging System. ${ }^{5}$ Since distant metastasis was detected, radiation therapy and chemotherapy were scheduled; however, approximately one week later the visual acuity of the left eye had decreased to 20/40, and there was increased proptosis $(5.5 \mathrm{~mm})$ and severe limitation of ocular movement (Fig. 3). Surgery was considered necessary to preserve vision and ameliorate the symptoms, therefore conservative 

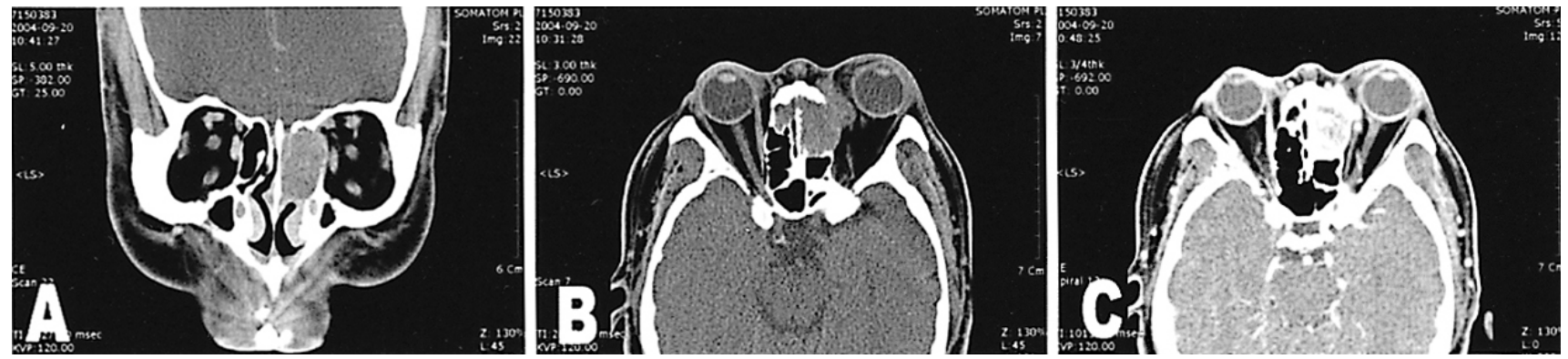

Fig. 1. Coronal (A) and axial (B,C) CT scans show a well-circumscribed, homogeneous mass in the left ethmoid sinus with extension into the left orbit and compression of the left medial rectus muscle. The tumor shows marked enhancement with contrast agents.
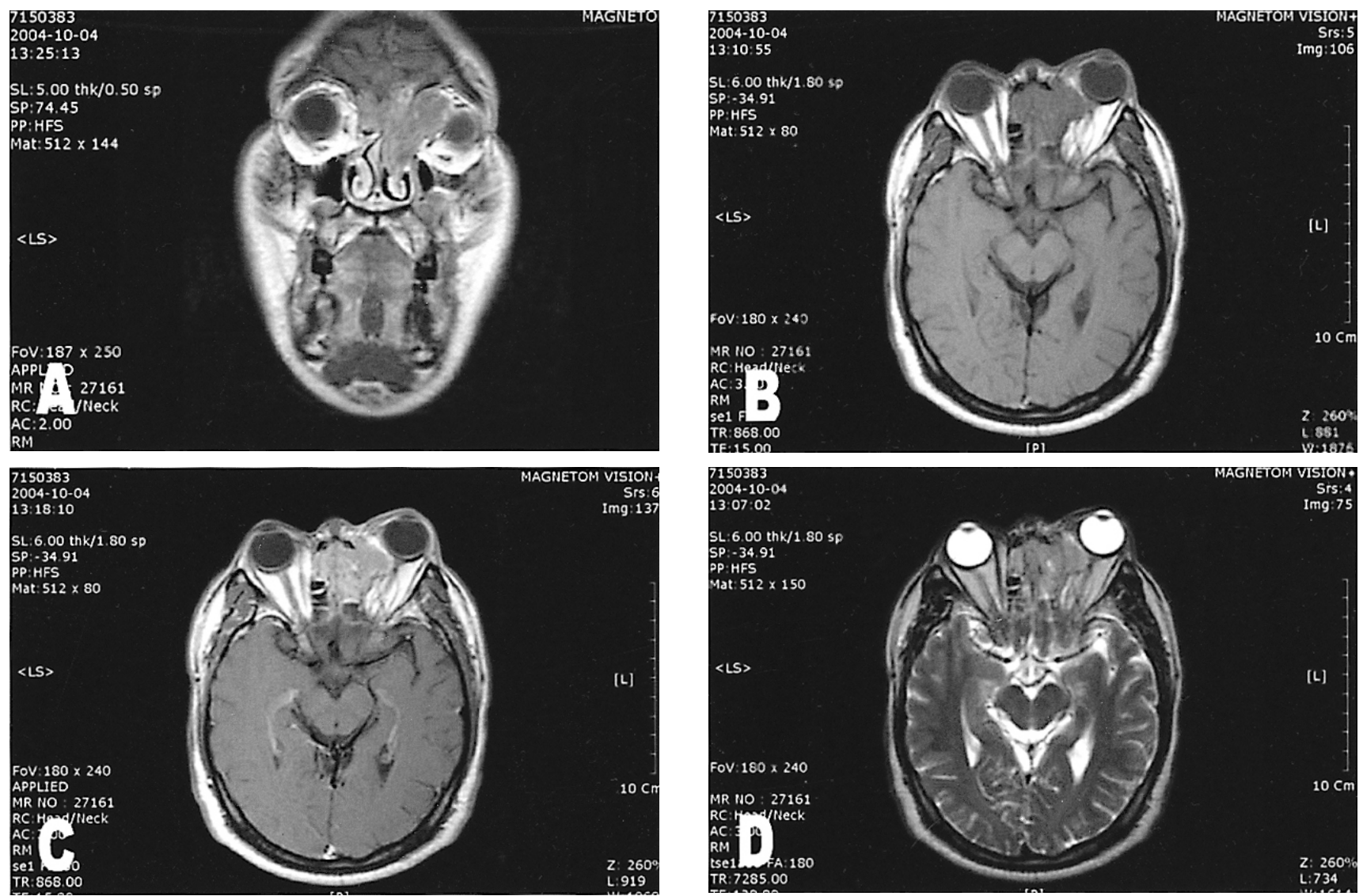

Fig. 2. Orbital magnetic resonance imaging (MRI) scans show rhabdomyosarcoma in left ethmoid sinus with low signal intensity on T1-weighted image (A,B) and moderate enhancement on enhanced scan (C). On a T2-weighted image, the lesion is isointense to extraocular muscles and hypointense to orbital fat (D). The lesion extends into the left orbit with compression of the left medial rectus muscle, and has intracranial extension with compression of the left side frontal lobe.

debulking and orbital wall decompression were performed through a transconjunctival incision in the inferior fornix under general anesthesia.

Gross examination revealed a well-circumscribed, ovoid, reddish tumor, which measured $1.5 \times 1.2 \times 1.0 \mathrm{~cm}$ (Fig. 4). Microscopic examination revealed diffuse small spindleshaped to round cells with abundant eosinophilic cytoplasm and eccentric nuclei. There was no evidence of crossstriation. Immunohistochemical analysis was positive for desmin, S-100, smooth muscle actin (SMA), and CD56, consistent with a diagnosis of alveolar RMS (Fig. 5). After surgery, the patient experienced some return of visual function and ocular motility (Fig. 6). The patient was treated with chemotherapy including adriamycin, combined with irradiation of the orbit and ethmoid sinus of $5800 \mathrm{cGy}$ over a period of 6 weeks. The chemotherapy was discontinued because the patient developed pancytopenia. A bone scan indicated progressive metastasis to the spine and the patient 


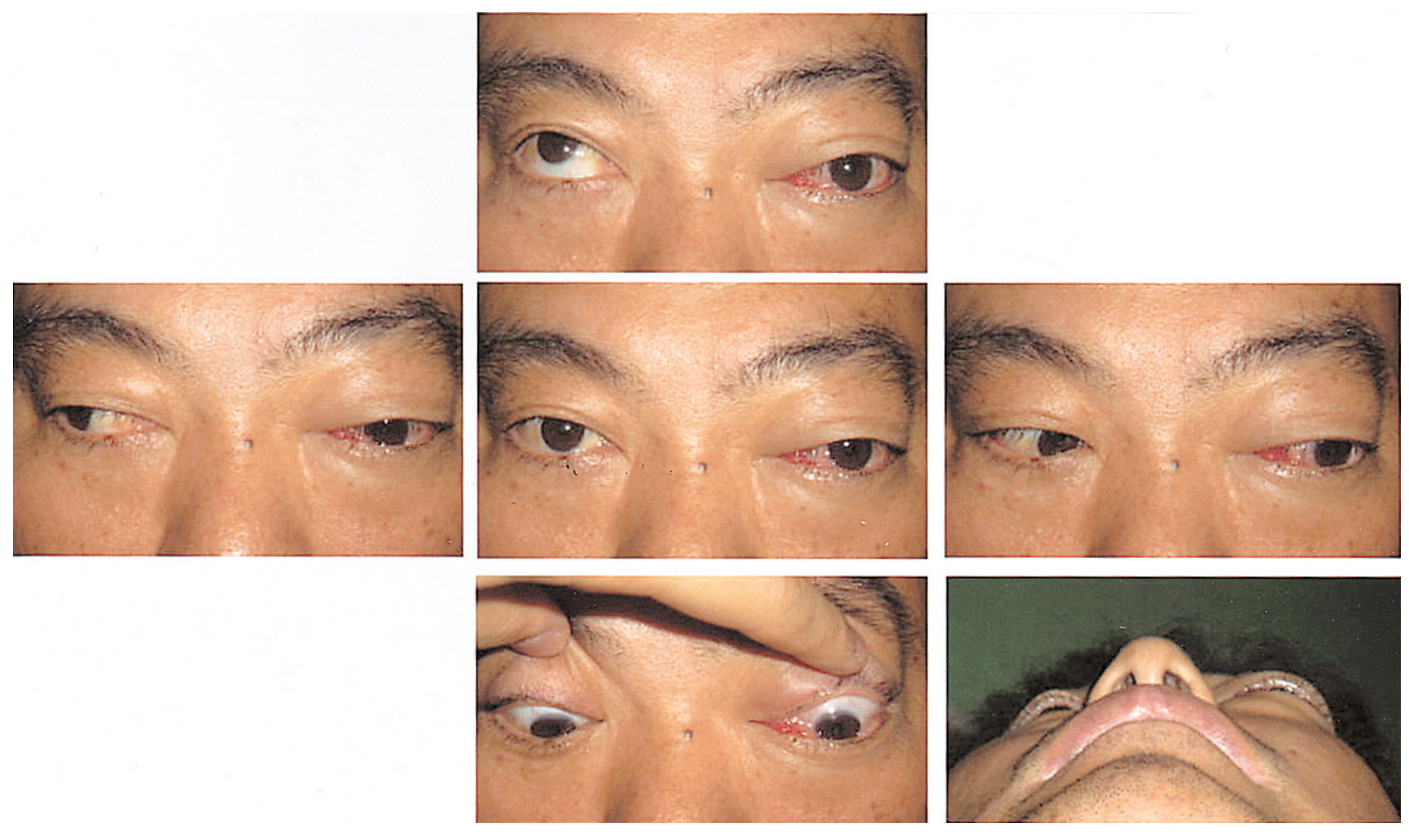

Fig. 3. Preoperative photography of a 48-year-old man suffering diplopia, with marked limitation of ocular movement, chemosis, proptosis in the left eye.

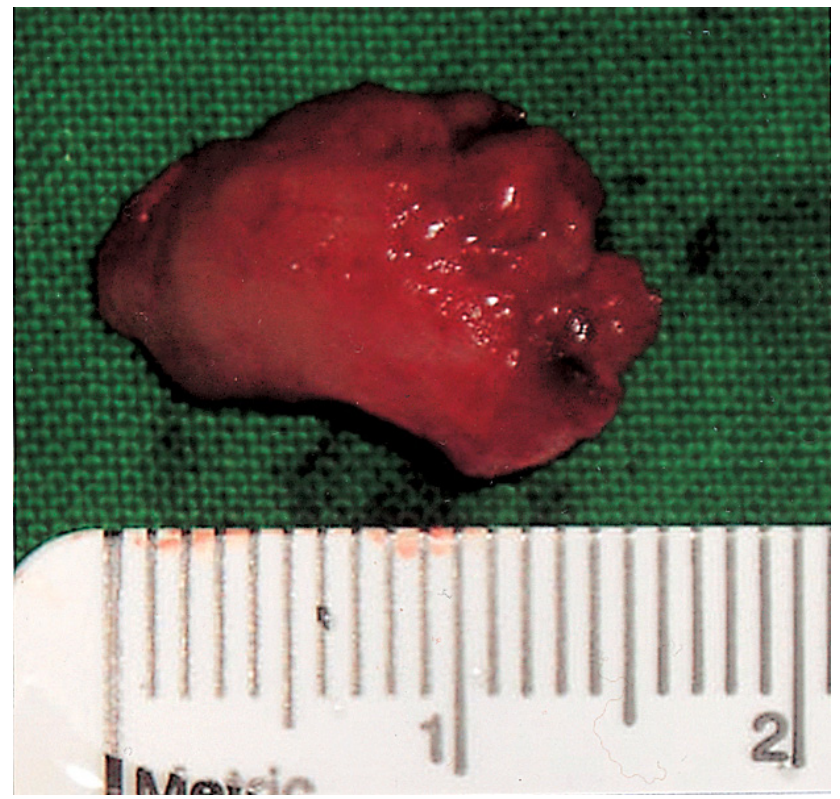

Fig. 4. Gross examination showed a circumscribed reddish tumor measuring $1.5 \times 1.2 \times 1.0 \mathrm{~cm}$.

died 7 months after the initial diagnosis.

\section{Discussion}

Although RMS is the most common sarcoma in the pediatric population, it is very uncommon in adults. ${ }^{1}$ Microscopically, the four major histopathologic types of RMS are: pleomorphic, embryonal, alveolar, and botryoid. ${ }^{6}$ In the Intergroup Rhabdomyosarcoma Study-IV, the overall distribution of the subtypes was $70 \%$ embryonal, $20 \%$ alveolar, and $10 \%$ others; ${ }^{5}$ however, Little et $\mathrm{al}^{7}$ reported subtype proportions in adults to be $43 \%$ pleomorphic, $34 \%$ embryonal, and $23 \%$ alveolar. The relative paucity of embryonal tumors and increase in alveolar and pleomorphic types are characteristic of adult RMS. ${ }^{8,9}$ Embryonal RMS is the most common variant found in the head and neck region while the alveolar and pleomorphic types are rare in the orbit. $^{1,6}$ The prognostic significance of this classification is undecided, but it is generally accepted that pleomorphic, better differentiated tumors have a better prognosis, while the alveolar subtype has the worst prognosis. ${ }^{10}$ Alveolar RMS occurs primarily in adults between the age of 10 and 25 years, and involves tumor cells that are mostly undifferentiated in nature, and therefore readily develop distant metastasis resulting in poor prognosis. ${ }^{11}$ Indeed, in the case presented here distant metastasis was already present at the time of diagnosis.

RMS shows a strong tendency for local invasion, local recurrence, and hematogenous and lymphatic metastasis. ${ }^{12}$ Alveolar RMS appears to be more aggressive that other subtypes and often shows lymph node involvement; therefore 

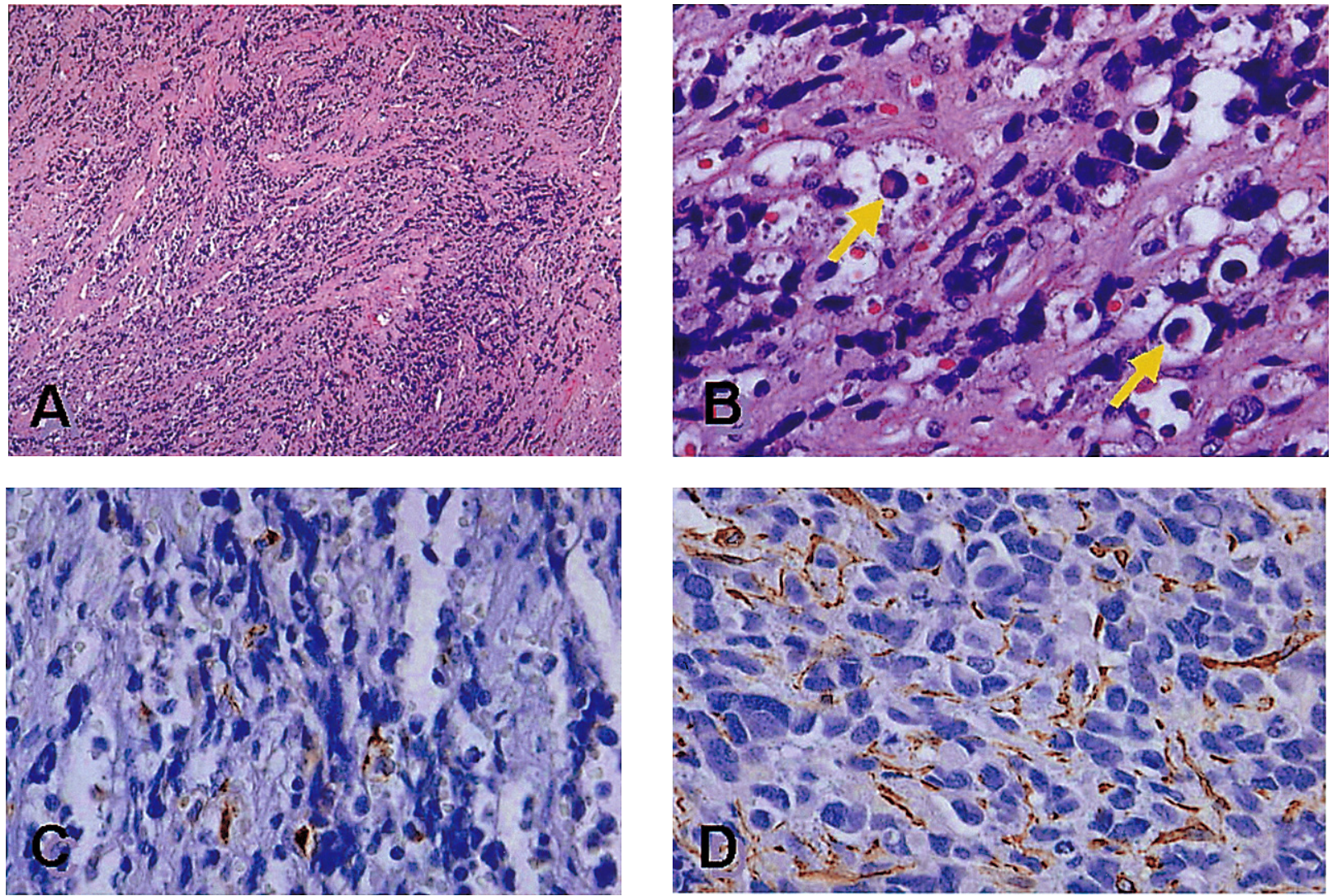

Fig. 5. Photomicrograph of isolated rhabdomyosarcoma. (A) Low magnification reveals diffuse small round cells (Hematoxylin-eosin, original magnification $\times 40$ ). (B) High magnification reveals round cells (rhabdomyoblast) with eosinophilic cytoplasm and eccentric nuclei (arrow) (Hematoxylin-eosin, original magnification $\times 400$ ). (C) Immunohistochemistry gave positive results with desmin, confirming the diagnosis of rhabdomyosarcoma (original magnification $\times 400$ ). (D) Immunohistochemistry with smooth muscle actin (original magnification $\times 400$ ).

combined treatment protocols involving chemotherapy and radiotherapy have typically been used. The most common pathway of distant spread of RMS is by hematogenous dissemination, particularly to the lung, cervical lymph nodes, and bone. ${ }^{6,13-15}$

Orbital RMS can present insidiously, clinically and radiologically mimicking other space-occupying lesions. The most characteristic presentations are rapid onset and progression of proptosis, and displacement of the globe. ${ }^{4}$ Intracranial extension and invasion of the paranasal sinuses are uncommon at presentation, while changes in the adjacent bone frequently occur. ${ }^{16}$ In the case presented here, the epicenter of the tumor was identified within the left ethmoid by $\mathrm{CT}$, and the medial rectus muscle was intact. It seems unlikely that the tumor originated in the orbit and then invaded and filled the ethmoid sinus since the presenting symptoms included proptosis that had been present for only 2 weeks. Therefore, our case was considered to have originated within the ethmoid sinus and subsequently invaded the orbit.

It is important that RMS tumors are distinguished from the more common tumors of the head and neck occurring in adults, including nasopharyngeal carcinoma, lymphoma, neuroendocrine carcinoma, melanoma, poorly differentiated carcinoma, and metastatic tumor. ${ }^{10,17}$ Differential diagnosis depends on imaging techniques in combination with histological and immunohistological analysis.

Various imaging methods have proved useful in the diagnosis and determination of the extent of RMS. CT and MRI are fundamental techniques for preoperative evaluation to determine tumor location and size, and are also important in evaluating residual or recurrent disease. ${ }^{4}$ In RMS, CT shows a moderately well-circumscribed, homogeneous, round to ovoid mass that is isodense to the extraocular muscle, which shows moderate to marked enhancement with contrast agents. On T1-weighted MR images, the tumor is usually isointense to the extraocular muscles and hypointense with respect to the orbital fat. On T2-weighted MR images, hypointensity, isointensity, and even hyperintensity may be appreciable with respect to both extraocular muscles and orbital fat. ${ }^{1,4}$

As differential diagnosis can be difficult when based solely on radiologic analysis, histologic evaluation is also required. Grossly, RMS is a well-circumscribed tumor while in the early stages. Larger, more aggressive tumors have an irregular border as a result of tumor invasion though the 


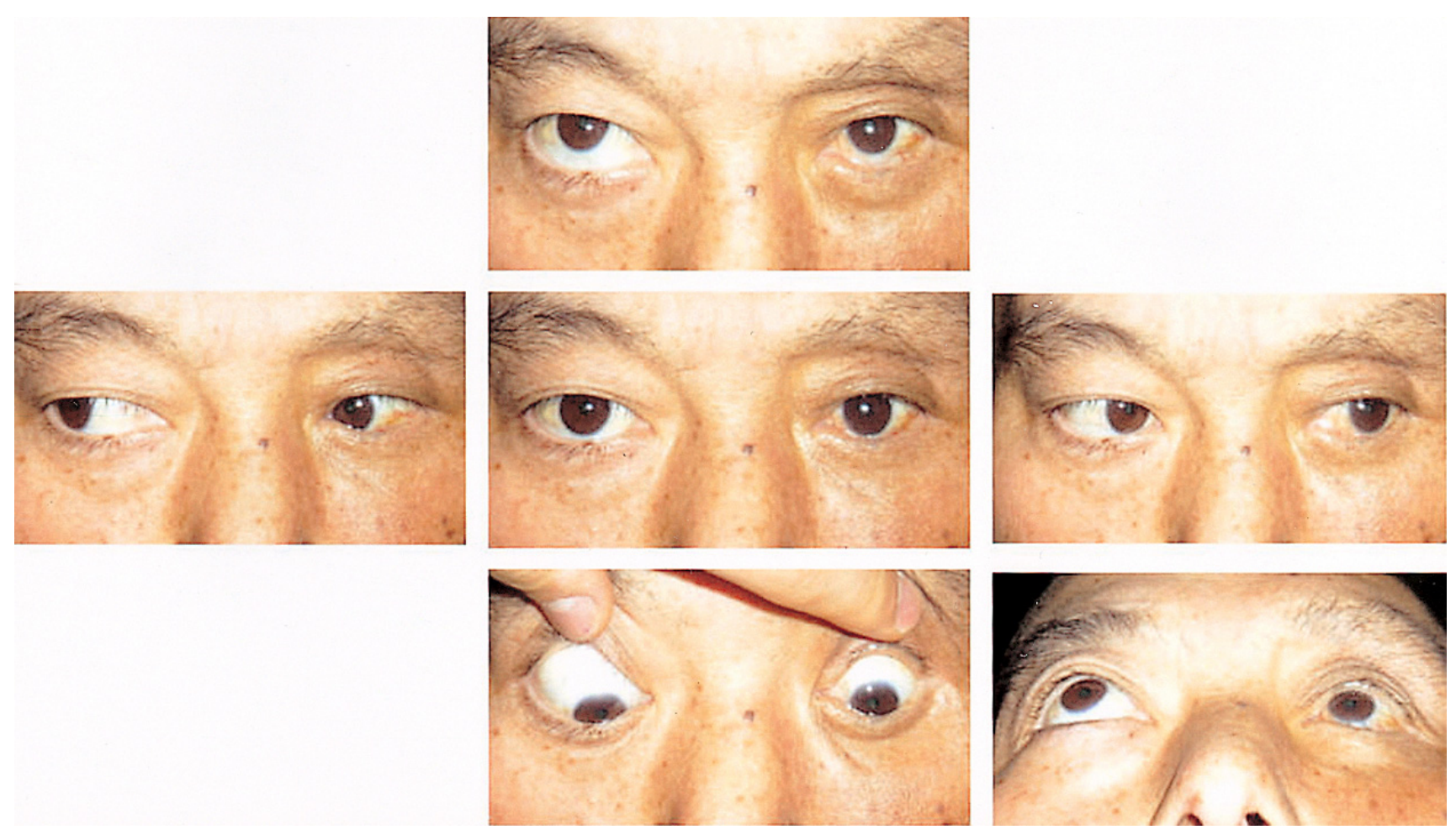

Fig. 6. 5-month postoperative photograph showing improvement of ocular movement and enophthalmos in the left eye.

pseudocapsule. The tumor has a light gray to pink color and may show areas of hemorrhage and cyst formation. Microscopically, RMS consists of small spindle-shaped to round rhabdoblasts with abundant eosinophilic cytoplasm and eccentric nuclei. The alveolar type appears as loosely arranged, malignant cells with septae that are reminiscent of the alveoli of the lung. ${ }^{1}$ Since these lesions exhibit morphological features in common with other spindle cell neoplasms further immunohistochemical analysis is necessary for definitive diagnosis ${ }^{1,9}$ RMS is characteristically positive for desmin, smooth muscle actin, and myoglobin.,13,14

Survival of patients with RMS generally depends on several factors, including histopathologic and cytologic tumor type, extent of disease at diagnosis, tumor burden at diagnosis, primary tumor site, patient age, cellular ploidy, and therapeutic response. ${ }^{12}$ The favorable prognostic characteristics for disease specific survival are: age $\leq 20$ years, tumor size $\leq 5 \mathrm{~cm}$, absence of regional or distant disease, surgical resection with negative margins, and pleomorphic type. 9,18

Treatment of RMS involves a combination of chemotherapy, radiation therapy, and surgery depending on the primary tumor site, pathologic findings, and the clinical stage of the tumor. ${ }^{4}$ For tumors that develop in the vicinity of the orbit a diagnostic biopsy is performed, and chemotherapy and radiation therapy are administered. In contrast, for sarcomas in the trunk, the extremities, and in the tissues adjacent to the testis, the tumor is surgically resected as aggressively as possible while avoiding local damage and preserving function, and chemotherapy and radiation therapy are administered. In cases where the tumor is too large for resection, surgery may be performed after the tumor size has been reduced by chemotherapy and radiation therapy. ${ }^{12}$

In summary, adult RMS is an extremely rare malignant tumor that is difficult to manage. A treatment plan should be made on an individual basis, taking into consideration the age of the patient, the disease stage, and the size of the tumor. Although it is rarely found in an adult, RMS should be considered in the differential diagnosis of orbital tumors irrespective of age. Immunohistochemisty should be performed in addition to radiologic imaging techniques and histology for a definitive diagnosis of RMS. Following diagnosis of RMS, systemic evaluation for distant metastasis is necessary.

\section{Reference}

1. Shields JA, Shields CL. Rhabdomyosarcoma: review for the ophthalmologist. Surv Ophthalmol 2003;48:39-57.

2. Crist W, Gehan EA, Ragab AH, et al. The Third Intergroup Rhabdomyosarcoma Study. J Clin Oncol 1995;13:610-30.

3. Lanzkowsky P. Rhabdomyosarcoma and other soft tissue sarcomas. In : Manual of Pediatric Hematology and Oncology, 3rd ed. New York: Academic Press, 2000; v. 1. chap. 20

4. Karcioglu ZA, Hadjistilianou D, Rozans M, DeFrancesco S. Orbital rhabdomyosarcoma. Cancer Control 2004;11:328- 
33.

5. Crist WM, Anderson JR, Meza JL, et al. Intergroup rhabdomyosarcoma study-IV: results for patients with nonmetastatic disease. J Clin Oncol 2001;19:3091-102.

6. Horn RC Jr, Enterline HT. Rhabdomyosarcoma: a clinicopathological study and classification of 39 cases. Cancer 1958;11:181-99.

7. Little DJ, Ballo MT, Zagars GK, et al. Adult rhabdomyosarcoma: outcome following multimodality treatment. Cancer 2002;95:377-88.

8. Esnaola NF, Rubin BP, Baldini EH, et al. Response to chemotherapy and predictors of survival in adult rhabdomyosarcoma. Ann Surg 2001;234:215-23.

9. Hawkins WG, Hoos A, Antonescu CR, et al. Clinicopathologic analysis of patients with adult rhabdomyosarcoma. Cancer 2001;91:794-803.

10. Friling R, Marcus M, Monos T, et al. Rhabdomyosarcoma: invading the orbit in an adult. Ophthal Plast Reconstr Surg 1994;10:283-6.

11. Michael C. Mesenchymal and fibro-osseous tumors. In : Stephen B, ed. Principle and Practice of Ophthalmic Plastic and Reconstructive Surgery, Philadelphia: W.B.
Saunders, 1996; v. 2. chap. 92

12. Shields CL, Shields JA, Honavar SG, Demirci H. Primary ophthalmic rhabdomyosarcoma in 33 patients. Trans Am Ophthalmol Soc 2001;99:133-42.

13. Porterfield JF, Zimmerman LE. Rhabdomyosarcoma of the orbit; a clinicopathologic study of 55 cases. Virchows Arch Pathol Anat Physiol Klin Med 1962;335:329-44.

14. Grieman RB, Katsikeris NF, Symington JM. Rhabdomyosarcoma of the maxillary sinus: review of the literature and report of a case. J Oral Maxillofac Surg 1988;46:1090-6.

15. Makishima K, Iwasaki H, Horie A. Alveolar rhabdomyosarcoma of the ethmoid sinus. Laryngoscope 1975; $85: 400-10$

16. Sohaib SA, Moseley I, Wright JE. Orbital rhabdomyosarcoma: the radiological characteristics. Clin Radiol 1998;53:357-62.

17. Koenigsberg RA, Noah R, Turtz A, et al. Rhabdomyosarcoma of the paranasal sinuses in an adult. Clin Imaging 1995;19:234-6.

18. Shields CL, Shields JA, Honavar SG, Demirci H. Clinical spectrum of primary ophthalmic rhabdomyosarcoma. Ophthalmology 2001;108:2284-92. 